\title{
Some ethical implications of The Human Genome Project
}

\author{
Charles J. Epstein, $M D$
}

What do we mean when we talk about the Human Genome Project? In its original formulation, this term referred to the organized efforts by the United States and other governments first to map and then to sequence the human genome, the ultimate objectives being to determine the linear sequence of the three billion base pairs that constitute the genome and to identify the individual genes that are coded for by this sequence. This goal is rapidly coming close to realization, although the actual identification of functional genes has proven to be a much more formidable problem than was perhaps initially appreciated. However, in the mean time, the overall concept of the human genome project has greatly broadened and expanded in many ways.

We now have a host of genome projects: projects to elucidate the mouse genome, the Drosophila genome, the yeast genome, the C. elegans (worm) genome, and the genomes of a vast host of microorganisms, both good and bad. Rather than just being interested in sequencing and gene identification, we now speak of functional genomics, which has the goal of finding how the individual genes actually function-how they produce their primary effects, how they are regulated, how they and their products interact. Given the complexity of biological interactions, functional genomics is virtually open-ended with regard to what it may encompass within the realm of biological investigation.

The development of new technologies makes it possible to envision radically new approaches to sequence determination, gene expression, and the identification of genes relevant to conditions of interest. In addition to the polymerase chain reaction (PCR), which is still the backbone of genomic investigation, we now have microarrays or chips, which make it possible to look at thousands of sequences simultaneously, and single-nucleotide polymorphisms, or SNPs, which will permit the detection of variation at virtually all, if not all, genetic loci.

Although the general goal of the human genome project is to discover the genes and to understand how they operate, it is not really possible to dissociate the description of the genome from the application of this information to matters of human health and disease. In fact, it was understood from the beginning,

From the Department of Pediatrics, University of California. San Francisco, Californat.

Presented at the annual meeting of the Western Society for Clinical mvestigation, the Western Association of Physicians, and the Western Section Americun Federation for Mcilial Re. scard, February 10, 2000, Carmel, California.

Charles 1. Epstein, MD, Department of Pediatrics. Box 0748. University of Califormia, San Francisco, CA 94143-0758.

Received: March 2, 2000.

Accepled: March 24, 2000. indeed, it constituted a major justification for the project, that what was learned would be applied in these areas. Hence, although not genome activities in a technical sense, the identification of and screening for disease genes, for susceptibility genes, and for polymorphic markers linked to such genes are subjects that are clearly identified with the genome project.

Finally, whereas the genome project was originally envisioned as a huge and ambitious undertaking for the public benefit and supported by public funds, it is clear that the private sector, the world of biotechnology, is playing an increasingly major and perhaps even a dominant role in determining how genome research and its applications to human health and disease will proceed. Therefore, while the public good is still in the equation, so is the corporate good, and that is ultimately defined in monetary terms.

\section{Presymptomatic diagnosis of late-onset disorders}

I shall discuss two issues related to the clinical and ethical implications of the project. The first, which is perhaps the most obvious clinical application of genomic information in current practice, is the presymptomatic diagnosis of late-onset genetic disorders. Two dominantly inherited conditions, Huntington disease and hereditary breast and ovarian cancer, constitute the paradigms for this approach, but they differ in quite important ways. In the case of Huntington disease, the diagnosis is certain (except for a small area of overlap between normality and disease), but it is not possible to accurately predict the time of onset and no effective therapy is available. By contrast, in the hereditary breast and ovarian cancer situation, the detection of a causative mutation does not constitute a certain diagnosis of cancer to come. Rather, it indicates that there is a significant risk or susceptibility of malignancy in the future, the magnitude of which may not be well defined. Although there are several possible approaches to prevention of malignancy, all have significant problems, and there is certainly no consensus on how to proceed.

Testing for both conditions has engendered an enormous amount of discussion with regard to the social and ethical issues that may be involved. These issues may be divided into five groups.

There are concerns about privacy and what might happen if the information becomes known to insurance companies and employers, to other members of the family, or to others in the community. The specific problems that have or might be encountered include discrimination, stigmatization, denial or increased costs of health and life insurance, and loss of jobs. The 
general term of "genetic discrimination" has been coined to cover these types of problems.

There are concerns about the presentation and interpretation of the test results. This applies particularly to the cancer testing situation in which accurate risk figures for particular mutations are often not available, and even if they are, only constitute probabilities and not certainties. Being able to communicate and deal with such information requires a certain degree of sophistication on the part of both the person transmitting the information and the person who is receiving it.

The lack of truly effective preventive measures for persons at risk of developing breast or ovarian cancer short of removal of the organs limits the options available to tested individuals who are found to be carrying mutations and places them in a quandary about how they ought to proceed. Again, the persons laying out the options need to be appropriately informed themselves.

The quality of the testing, both in technical and procedural terms, is of concern. Included here are issues surrounding laboratory procedures: reagents, controls, and the like, and the information provided to health professionals and to those being tested both before and after the testing is performed. In light of all of the preceding points, it is clear that there are many things that the patient interested in testing needs to understand before the testing is done and much that needs to be communicated and discussed after the testing is completed.

Finally, a matter of concern is the commercialization of testing. As new genetic tests potentially applicable to a significant portion of the population come into view, they are very often developed, patented, and performed by commercial laboratories affiliated with ever larger biotechnology/pharmaceutical companies. Although the major investment that goes into cloning the relevant genes and piloting the testing should not be minimized, it must still be realized that these companies are expected to cover their developmental costs and to give a nice return to their investors. Therefore, it is clearly in their interest to promote or market the tests as widely as possible and to charge enough to make them profitable. Given all that I have just said about the many concerns about testing, there are many potential areas of conflict around the marketing of tests. And, even if all of these issues were resolved, then the lack of availability of the test to all who might qualify but might not be able to afford it becomes an ethical problem in its own right.

Although some of the concerns I have just outlined might not seem to be ethical issues per se, they are readily translated into ethical problems. For example, is it ethical to offer a test for which there are no or only limited therapeutic options, or for which the risk figures are still uncertain or which might lead to loss of insurance? Because of all of these issues, privacy, procedural matters, interpretation of the results, availability of options, and commercialization of testing, it has been suggested that presymptomatic genetic testing is fraught with problems and, in the extreme, may actually be dangerous to those being tested. As a result, there have been calls in many quarters for limiting testing, for considering presymptomatic genetic testing as experimental and therefore subject to human experimentation guidelines, for guidelines and voluntary regulation by professional organizations, and for more stringent governmental regulation. Many organizations and committees have dealt with these issues, and a variety of regulations, sometimes quite restrictive, have been suggested although not implemented.

My general philosophical or, if you will, ethical approach, to Huntington disease and breast and ovarian cancer testing is that people, if they are appropriately prepared to receive it, are entitled to accurate information about their genetic status, however uncomfortable the knowledge might turn out to be. The preparation is crucial, and it takes time. However, these conditions, which are highly penetrant and essentially monogenic in inheritance, represent just the tip of the iceberg of the type of testing that we are being told that the genome project is going to make possible. The operative concept in what is promised for the future is complex trait analysis for susceptibility genes and quantitative trait loci or QTLs. It is becoming ever more apparent that the human genome is replete with variation that extends down to the level of single nucleotides, the SNPs. Although some proportion of this variation may have absolutely no effect, much of it undoubtedly contributes to the host of structural, physiologic, and psychological differences that distinguish one human being from another and to different liabilities or susceptibilities to the development of virtually any disease or disorder that one might think of.

\section{Susceptibility factors}

Considerable effort is now being expended to search out the genetic components or susceptibility factors underlying such common conditions as diabetes, coronary artery disease, allergic and autoimmune disorders, Alzheimer disease, bipolar disease, and schizophrenia. To date, the going has been very slow, but it is hoped that the new technologies, especially microarrays, will accelerate the process. Why are we so interested in these susceptibility factors and QTLs? The immediate reason is to understand their contribution to the pathogenesis of the disorders in question and, with that understanding, hopefully to be able to devise rational therapeutic approaches. However, another reason that has been put forward again involves the issue of presymptomatic diagnosis, and I would like to quote from a recent article by Francis Collins ${ }^{1}$ in which he presents a "Hypothetical Case in 2010." The gist of this hypothetical case is the following:

John, a 23 year-old college graduate. . . [with] a serum cholesterol level of $255 \mathrm{mg}$ per deciliter. . [ [and] a strong paternal history of myocardial infarction, agrees (and signs informed consent) to undergo 15 genetic tests that provide risk information for illnesses for which preventive strategies are available. He decides against an additional 10 tests involving disorders for which no clinically validated preventive interventions are yet available....

John is pleased to learn that genetic testing does not always give bad news, his risks of contracting prostate cancer and Alzheimer's disease are reduced, because he carries low-risk variants of the several genes known in 2010 to contribute to these 
illnesses. But John is sobered by the evidence of his increased risks of contracting coronary artery disease, colon cancer, and lung cancer. Confronted with the reality of his own genetic data, he arrives at that crucial "teachable moment" when a lifelong change in health-related behavior, focused on reducing specific risks, is possible. And there is much to offer... pharmacogenetics. .. annual colonoscopy. .. support groups. This vision of genetically based, individualized preventive medicine is exciting, and it could make a profound contribution to human health. ${ }^{1}$

Collins cast this hypothetical case in very narrow terms, restricting himself to conditions for which, as he put it, "preventive strategies are available." However, we already know from the Huntington disease example that people are willing to seek information on conditions for which no such therapeutic strategies exist. Given the numbers of sequences that can be packed on a single chip, it is certainly questionable in my mind whether the chip-makers would limit the contents of their chips only to preventable conditions and whether the public would not seek broader testing. But even leaving this aside, one might ask how realistic or desirable the Collins scenario is. Here is a summary of what one knowledgeable dissenter, Angus Clarke, ${ }^{2}$ a Welsh geneticist, has to say:

The fact that so many genes and nongenetic factors are involved in the etiology of these common diseases means that identification of inherited predisposition is of little use at the individual level; it will never be possible to predict those who will be affected nor to know when an individual will develop a disease if he does so at all....

Such tests will not be predictive; they will only convey information about relative risk, and will leave as much uncertainty as they are likely to resolve. Furthermore, however carefully the risk information is conveyed, very important issues will remain about how the information is understood and used by the individual. ... .

Such tests may nevertheless be developed for a combination of reasons: commercial "necessity" in the biotechnology and insurance industries, professional enthusiasm among clinicians and research scientists, and the desire in all of us to apply knowledge to important questions of human health.... While susceptibility screening may be bad science, it is likely to be excellent business....

The use of DNA technology to identify those with better or worse health prospects, a genetic sorting into sheep and goats, could create social inequalities, or more likely be used to reinforce existing inequalities... This notion of a genetic underclass resonates with the echoes of eugenics from earlier this century....

The final disadvantage of genetic susceptibility testing. . . is that it promotes medicalization and genetization. The medicalization of life will be promoted because of the large number if individuals who will be identified as being at increased risk of something. Genetization will also be promoted; this is the explanation of differences between individuals and groups in purely genetic terms, and the consequent overemphasis of genetic factors in planning the provision of health care. ${ }^{2}$
Some, perhaps all, of Clarke's arguments may be extreme, I present them in some detail because they focus on many of the relevant issues-difficulty in conveying and interpreting risks, social inequalities, eugenics, medicalization, genetizationthat form the basis for the extensive ethical debate that is swirling around the genome project. They also bring up issues that have not been fully explored, such as the likelihood of being able to identify major susceptibility factors that can offer meaningful opportunities for intervention in complex disorders. This point is raised again below with regard to the identification of genes that affect cognition.

Where does the truth lie? My opinion is that it lies somewhere between what Collins finds "exciting" and Clarke finds unacceptable. However, I do believe with Clarke that there will be increasing pressure to develop and market tests and that it will be increasingly more difficult, for both logistic and economic reasons, to provide the pre- and post-testing counseling that will be required to ensure that people know what they are getting into and what the results actually mean. I do not have great confidence in the willingness of commercial enterprises to limit the scope of testing in which they are heavily invested.

In so far as test promotion is concerned, the dividing line between a limited number of significant susceptibility factors, knowledge of which, as Collins suggests, might be beneficial, and the potentially far greater number of minor ones that could be tested for, will become very fuzzy indeed. The broader ethical issues of medicalization and genetization and of creating or reinforcing social inequalities cannot be disregarded out of hand, but I am not sure that they constitute strong arguments against testing in a limited and defined manner.

\section{Genetics of cognition}

I now come to the second broad issue I wish to discuss. The same approaches being taken to discover disease susceptibility genes can, although possibly with considerably more effort in some instances, be used to look at the genetic basis of traits that we do not equate with disease or its treatment, traits such as physical appearance, stature, behavioral and emotional characteristics, and cognitive ability. If you read any of the futuristic writings written for popular consumption, these are the possibilities that most capture the writers' and apparently the public's imaginations. Carried to the ultimate, such knowledge, coupled with current and easily foreseeable reproductive technology, would enable us to predict, indeed, to choose, the traits of our children. However, rather than getting caught up in the already extensive debate about the future, I would like to consider just one point that has already excited and will continue to excite considerable controversy: the genetic analysis of cognitive ability or intelligence. The issue here is a very critical one: are there traits, such as cognitive ability, that should not be subjected to genetic analysis?

The arguments for and against searching for quantitative trait loci involved in general cognitive ability, referred to as $g$, were recently presented by Robert Plomin, ${ }^{3}$ a proponent of such investigations. Again, I present them in some detail to point out what the relevant scientific and genetic issues are: 
There is considerable consensus among scientists, even those who are not geneticists, that $g$ is substantially heritable. . Estimates based on the entire body of data make the [heritability] about $50 \%$, indicating that genes count for about half the variance in $g .$. The data that provide evidence for a genetic effect on $g$ also provide the best available evidence for the importance of environmental factors that are independent of genetics.

Genes associated with $g$ will be identified. . [but] it is likely that the average size of effect of each individual gene is small for complex traits, perhaps the individual gene on average will account for less that $1 \%$ of the variance, with few effect sizes larger than $5 \%$.

The ultimate scientific goal is not just finding the genes but understanding how they function.. Finding genes associated with $g$ and other cognitive abilities and disabilities in humans will provide discrete windows through which brain pathways leading from genes to complex cognitive processes of learning and memory can be observed... [and] to lead to gene-based diagnoses and treatment program for mild mental retardation, and clarification of its overlap with learning disabilities.

No policies necessarily follow from finding genes associated with $g$, because policy involves values. . Public policy does not necessarily follow from scientific findings... The only think that seems completely clear is that nothing will be gained by ignoring the issue and pretending that $g$ does not have a significant genetic component. ${ }^{3}$

After arguing for the importance of studying the genetic basis of $g$, Plomin ${ }^{3}$ then deals with the concerns. Without going into his specific responses, here are some of the issues he raises:

Will a DNA chip for $g$ make the 1997 science fiction film GATTACA, in which individuals are selected for education and employment on the basis of their DNA, come true?

Is it possible that there are parents who would use DNA chips for $g$ prenatally for eugenic purposes?

Will DNA chips for $g$ be used for postnatal screening to enable interventions that avoid risks or enhance strengths?

... average differences between classes and ethnic groups. . .

... knowledge about genetics might change attitudes. . .

... finding genes associated with $g$ will undermine support for social program because it will legitimate social inequality as "natural"3

Finally, having raised all of these issues, for which he has answers, Plomin' $\mathrm{s}^{3}$ bottom line is the following:

There is fear lurking in the shadows that finding genes associated with $g$ will limit our freedom and our free will. In large part such fears involve misunderstandings about how genes affect complex traits like $g$. Finding genes associated with $g$ will not open a door to Huxley's brave new world where babies are engineered to be alphas, betas, and gammas. The balance of risks and benefits to society of DNA chips for $g$ is not clear... What is clear is that basic science has much to gain from functional genomic studies of brain function associated with learning and memory. We need to be cautious and to think about societal implications and ethical issues. But there is also much to celebrate here in terms of the increased potential for understanding our species' unparalleled ability to think and learn. ${ }^{3}$

As would be expected, the opposition (again as represented by Clarke $e^{4}$ has had much to say about all of this, but I want to quote only the most trenchant arguments:

In addition to the potential harmful effects of promoting racism or the institutional misapplication of such research... the genetic dissection of intelligence implicitly but powerfully conveys two contentious messages: (i) that people's genetic constitution determines their abilities and personal characteristics; (ii) that knowledge of a person's IQ tells is of central importance to that person's worth or value. Researchers in this field will rightly repudiate such crude notions, but "actions speak louder than words"; whatever the personal views of those conducting the research, the research will of itself convey these messages. My judgment is that the potential for abuse of such research [on IQ] should lead us to concentrate our research efforts elsewhere. ${ }^{4}$

I do not agree with this recommendation and am very leery about the wholesale proscription of whole areas of research, whether they involve cognition or cloning, yet another contentious area. I believe with Plomin that there are valid reasons for investigating the genetic basis of cognition, although, as he clearly indicates, being able to identify genes of significant effect is very much a gamble. However, the potential implications of identifying such genes cannot be ignored, and I would echo the comments of Peter Harper, ${ }^{5}$ another Welsh geneticist:

For an attribute regarded as so important by both society and many families, I do not think that. . . a conclusion. . . that the work is unlikely to form the basis of future tests of prediction, because of the probable small effect of individual loci. . . can be taken for granted; there could well be pressure to apply (or misapply) any marker or series of markers found to be relevant, even without an adequate scientific basis for doing so.. I do not consider this research necessarily to be unethical, but there are undoubtedly major and controversial issues involved, and I think that it is unethical to undertake the work without giving these the fullest consideration. ${ }^{5}$

I like Harper' $\mathrm{s}^{6}$ statement, because it articulates the type of consideration in which I believe we all must engage to prepare both ourselves and the public for what is going to come. Furthermore, I believe that we must inform the public about what we are doing and why we are doing it. In doing so, I do not think that we do ourselves any good in dismissing uncomfortable questions by falling back on the argument that scientific knowledge is ethically neutral, that it is all right to do whatever we can do, and that it is only the application of knowledge that leads to ethical problems. As strongly as I support intellectual and aca- 
demic freedom, such an argument smacks too much of the kind of statements made by German scientists when asked about their research prior to and during World War II. ${ }^{6}$

\section{Conclusion}

If we are in fact going to be able to go on with research in such sensitive areas as presymptomatic genetic testing, genes involved in cognition, embryo selection, gene therapy (germline and otherwise), and human cloning, we need to make sure in our own minds that we are doing the right thing. This becomes ever more important as the power of our analytical and manipulative technology touches on the human fears and concerns that conjure up the Frankenstein and Brave New World and Jurassic Park myths. As the raging disputes about genetically modified crops-Frankenfoods as the activists term them-clearly demonstrate, we ignore these fears and concerns at our own peril. Therefore, it behooves us to anticipate and confront these concerns, ethical and social, in a deliberate, rational, sensitive, and constructive manner. If we do, then there is at least a good chance that the great promise of the Human Genome Project for benefiting mankind will ultimately be realized.

\section{References}

1. Collins FS. Shattuck Lecture: Medical and societal consequences of the Human Genome Project. N Engl J Med 1999;341:28-37.

2. Clarke AJ. The genetic dissection of multifactorial disease: The implications of susceptibility testing. In: Harper PS, Clarke AJ, editors. Genetics, society and clinical practice. Oxford: Bios, 1997:93-106.

3. Plomin R. Genetics and general cognitive ability, Nature 1999;402(Suppl):C25-C29.

4. Clarke AJ. Limits to genetic research? Human diversity, intelligence and race. In: Harper PS, Clarke AJ, editors. Genetics, society and clinical practice. Oxford: Bios, 1997:207-218.

5. Harper PS. Genetic research and "IQ." In: Harper PS, Clarke AJ, editors. Genetics, society and clinical practice. Oxford: Bios, 1997:201-205.

6. Paul DB, Falk R. Scientific responsibility and political context: The case of genetics under the swastika. In: Maienschein J, Ruse M, editors. Biology and the foundation of ethics. Cambridge: Cambridge University Press, 1999:257-275. 